\title{
Übersetzen als kulturelles Handeln: Zur politischen Motivation der Übersetzungsstrategien in Thomas Braschs Shakespeareübersetzung "Wie es euch gefällt"
}

\begin{abstract}
This article analyses the different translation strategies in Thomas Brasch's German translation of Shakespeare's As You Like It with respect to their impulse towards adaptation. The article takes as its starting point the assumption that the cultural performance of a translation can be experienced independently of the criterion of similarity. This is the case whenever one manages to elucidate how a translation cites and at the same time dislocates its source and target (con-)texts. In this double distance between source context and the inherited context of its own target language translation is manifested as an act of cultural intervention. Brasch's analysed translation reveals its emotional, linguistic and cultural richness as the reader recognizes how the various translation strategies are meaningfully related to his political convictions.
\end{abstract}

Keywords: Translation as manipulation; Cultural Studies; Reception; Brasch.

Zusammenfassung: Der folgende Artikel untersucht die sich in Thomas Braschs Übersetzung von Shakespeares „Wie es euch gefällt” manifestierenden Übersetzungsstrategien im Hinblick auf den ihnen jeweils zugrundeliegenden

Der Autor ist Professor an der Universidade do Estado de Santa Catarina. 
Bearbeitungsimpuls. Der Artikel geht dabei von der Annahme aus, dass die kulturelle Leistung einer Übersetzung als unabhängig von Ähnlichkeitskriterien erfahrbar werden kann; nämlich dann, wenn es gelingt, zu verdeutlichen, wie sie ihre beiden an Ursprungs- und Zielsprache gebundenen kulturellen (Kon-)'Texte als sprachlich-ästhetisch gefasste Welten zitiert und immer zugleich variiert. In dieser doppelten Distanz zu Ursprungstext und tradiertem Kontext der eigenen Zielsprache manifestiert sich Übersetzung als kulturelle Handlung. Braschs Übersetzung enthüllt ihren emotionalen, sprachlichen, und kulturellen Reichtum, sobald der Leser erkennt, wie die politische Überzeugung des Autors seinen verschiedenen Übersetzungsstrategien zugrundeliegt.

Stichwörter: Übersetzung als Manipulation; Cultural studies; Rezeption; Brasch.

Resumo: $\mathrm{O}$ artigo analisa as diferentes estratégias de tradução usadas por Thomas Brasch na sua tradução alemã de "As You Like It" de William Shakespeare, especialmente quanto aos estímulos à adaptação do texto de partida. O artigo parte da pressuposição que se pode experimentar o valor cultural de uma tradução independentemente de critérios de fidelidade, ou seja, quando se consegue mostrar como uma tradução cita e simultaneamente varia os seus dois (con-)textos culturais. Nesse duplo distanciamento para com o contexto de partida e o contexto da própria língua alvo, manifesta-se a tradução enquanto um ato de intervenção cultural. A tradução de Brasch revela a sua riqueza emocional, lingüística e cultural a partir do momento em que o leitor percebe de que forma a convicção política do autor fundamenta as várias estratégias de tradução.

Palavras-chave: Tradução enquanto manipulação; Estudos culturais; Recepção; Brasch.

\section{Einleitung}

Angesichts der auch linguistischen Notwendigkeit, Äquivalenz als eine geregelte Differenz zu definieren, ${ }^{1}$ scheint mir Walter BenjAmIN ohne Zweifel

1 Vgl. den grundlegenden Aufsatz von JAKoBson (2000). 
Richtiges zu treffen, wenn er festhält, dass die Größe einer literarischen Übersetzung ${ }^{2}$ nicht ,in der oberflächlichen und undefinierbaren Ähnlichkeit zweier Dichtungen” besteht, ja dass nicht einmal „Übersetzung möglich wäre, wenn sie Ähnlichkeit mit dem Original ihrem letzten Wesen nach anstreben würde" (BENJAMIN 2001: 196). Wenn eine Übersetzung nicht in erster Linie (linguistische und kulturelle) Ähnlichkeit anstrebt, was kann sie dann dem Kritiker oder Analytiker als Untersuchungsmaterial anbieten?

Benjamins Verständnis repräsentiert insofern einen wichtigen Ansatz, als er die rhetorische und semantische Distanz zwischen Ursprungstext und Übersetzung nicht nur für notwendig hält, sondern eben gerade darin die Möglichkeit eines originären Erkenntnisgewinns des Lesers sieht. Dies legt nahe, Übersetzen als kreativen Akt zu verstehen, weshalb für das jeweilige Textprodukt die dieses erhellenden Regeln jeweils erst gefunden werden müssen. Diese können nicht allein und nicht einmal in erster Linie aus dem Ausgangstext und seinem Kontext gewonnen werden. ${ }^{3}$ Der Wert einer bestimmten Übersetzung ist dann darin zu suchen, wie sie ihre beiden an Ursprungs- und Zielsprache gebundenen kulturellen (Kon-)Texte als sprachlich-ästhetisch gefasste Welten zitiert und immer zugleich variiert und verschiebt, also an ihrer Distanz zum Ursprungstext wie zum tradierten Kontext der eigenen Zielsprache. In dieser doppelten Distanz wird die Übersetzung als kulturelle Handlung erfahrbar. Dabei verstehe ich als das Zitathafte einer Übersetzung den Aspekt an ihr, der Merkmale des Ausgangstextes nachbildet, also deren Bedeutung für die eigene Aussageabsicht innerhalb der Zielkultur anerkennt, während der Zusatz Variation oder Verschiebung auf die Momente der Übersetzung verweist, von denen

2 Ist im folgenden von Übersetzung die Rede, so beziehe ich mich auf Übersetzungen literarischer Texte.

In einem historisch vergleichenden Essay zu archaisierenden und verjüngenden Übersetzungsstrategien französischer Hamletübersetzungen kommt DÉPRATS (2004: 78) zu dem Schluss, "the truth of the translation must be envisaged not in terms of adequacy, but in terms of manifestation." Übersetzungen gerecht zu werden, heißt ihre Strukturen als legitimen Ausdruck eines eigenen Gestaltungswillens anzuerkennen, den es zuallererst zu verstehen gilt. 
diese Autorität verworfen wird. Gleichzeitig vermittelt diese Vorstellung des variierenden Zitats zwischen Ursprungstext und Übersetzung und ihren jeweiligen kulturellen Kontexten. Sie vermeidet also interpretatorischen oder übersetzerischen Narzissmus, ohne den in einer Übersetzung zweifellos vorhandenen egozentrischen Impuls (ihr Interesse, im Anderen des Ausgangstextes teilweise Eigenes zu finden, zu betonen und einzuschreiben) leugnen zu wollen. ${ }^{4}$

Diese Konzeption scheint mir auch Thomas Braschs Übersetzung von Shakespeares As You Like It angemessen, die ihren in diesem Sinne hybriden Charakter bereits durch einen Hinweis auf dem Buchtitel ausweist. Dort heißt es nämlich: „übersetzt und bearbeitet von Thomas Brasch." Einem Bearbeiten liegt aber ein individueller Deutungshorizont zugrunde, der in seinen ideologischen Einschreibungen historisch-kulturell und also sozial gebrochen ist. ${ }^{6}$ Die Einsicht in die fundamentale Bedeutung dieser ideologischen Dimension beim Übersetzungsprozess ist

4 Zum Konzept der Übersetzung als Ausdruck eigennütziger Interessen der Zielkultur, siehe insbesondere Aaltonen (2000) und Venturi (1995). Philip Leivis Konzept der „abusive fidelity” (in Venuti 1995: 23/24) scheint mir dabei eine ähnliche Absicht zu verfolgen wie meine Vorstellung der Übersetzung als ein variierendes Zitat, nämlich Übersetzung als Manipulation eines sprachlich gefassten kulturellen Traditionsrahmens zu verteidigen. Eine eingehende, kritisch vergleichende Darstellung dieser Konzepte kann im Rahmen dieses Aufsatzes aber leider nicht geleistet werden.

5 Die Übersetzung erschien zuerst 1993 im Insel-Verlag unter dem Titel William Shakespeare. Wie Es Euch Gefällt. Übersetzt und bearbeitet von Thomas Brasch, und wurde 2002 im Sammelband Thomas Brasch Shakespeareübersetzungen wiederaufgelegt. Auch der zweite Titel behält die Spannung zwischen Übersetzung und Bearbeitung bei, indem er Thomas Brasch als Autor ausweist und so die Hierarchie zwischen "Original" und Übersetzung unterläuft.

$6 \quad$ Eine die Äquivalenzproblematik und damit die Legitimationsfrage berücksichtigende Beschreibung, was eine Bearbeitung ausmacht, findet sich bei AALTONEN (2000: 64): "An adaptation as a translation strategy intersects the source text at what is perceived to be the essence of it [...]. An adaptation may thus reactualise the source text by translating only parts of it, while other parts vanish [...], but in all cases the adaptation still claims to represent the source text in the target system." Genau dies ist auch Braschs Vorgehensweise (siehe 2.1). 
auch für textorientierte Ansätze nicht neu. ${ }^{7}$ Sie legt nahe, dass man Braschs Shakespeareübersetzungen zwar mit den üblichen Kategorien der ,aneignenden”, ,verfremdenden” oder „vermittelnden” Übersetzung im Sinne Goethes, Schleiermachers oder Drydens ansatzweise beschreiben, aber ihren Bearbeitungsimpuls und damit die Funktion dieser Strategien nicht erklären kann. Dieser Impuls liegt, und das ist einer der grundlegenden Ansätze dieser Arbeit, in BRASCHs spezifischer, ästhetischer und politischer Sensibilität, in einem - wie BRASCH selbst es ausdrückt - „großen Thema”, das er für seine eigene Person andeutet als den Konflikt ,zwischen hartnäckig auf ihrer Individualität beharrenden Kreaturen und einer zunehmend versteinernden Warenwelt” (BRASCH 1978: 40).

\section{Thomas Brasch Shakespeareübersetzung Wie es euch gefällt}

Braschs betrachtete seine Shakespeareübersetzungen keineswegs als Gelegenheitsarbeiten. Sie galten ihm als integraler Bestandteil seiner schriftstellerischen und kreativen Arbeit, wie man aus diesbezüglichen Reflexionen ablesen kann, die er in Interviews und Selbstzeugnissen darlegte. Denn das sogenannte große Thema beeinflusst Braschs symbolische Aneignung der eigenen Umwelt und Zeit, nicht nur die Aneignungen historisch vergangener Kulturen. Brasch ist ein produktiver Rezipient, nicht nur der Literatur anderer, sondern auch - in der eigenen Literatur und durch seine Figuren - der eigenen Lebensverhältnisse.

\footnotetext{
So etwa in der von Nord im Anschluss an Vermeer favorisierten Skopos-orientierten Übersetzung (NORD 1991: 24-26/32-35). Die intendierte Aussageabsicht des Zieltextes innerhalb seiner eigenen kommunikativen Situation ist in erster Linie an die Person des Übersetzers (bzw. seines Auftraggebers) gebunden und bestimmt als solche die Übersetzungsstrategien. Allerdings bindet Nord in ihren Beispielen den Skopos des Zieltextes dann doch wieder an einen mehr oder weniger vage analysierten bzw. inferierten Skopos des Ausgangstextes. So verdeckt sie die ideologische Komponente bei der Konstruktion des Skopos des Ursprungstextes. Diese auf Skopos-Äquivalenz ausgerichtete Vorgehensweise mag bei pragmatisch orientierten Texten noch angemessen sein, aber sie entspricht nicht der kommunikativen Funktion eines ästhetischen Textes, der ja gerade keine eindeutig pragmatisch definierte Aussageabsicht hat.
} 


\subsection{Der Übersetzer als produktiver Rezipient}

BRASCH bekennt als Bedingung eines für ihn geglückten Übersetzens in einem Gespräch mit Martin LiNZER kurz nach der Erstaufführung seiner Übersetzung Wie es euch gefällt am Berliner Schillertheater 1993 folgendes (LINZER 1993: 28):

Man kann auch etwas gewinnen, hinzufügen, wenn man die Struktur, die Temperatur, die politische Poesie nicht verletzt. Es ist wichtig, sich in etwas Fremdem wiederzufinden, ohne es auf falsche Weise bekannt machen zu wollen. [...] Ohne die Zeit zu verschieben, in der ich lebe, muß ich einen Weg finden oder zu finden versuchen, Shakespeare für mich verständlich zu machen. Soviel wie möglich für mich nutzbar zu machen. Sowenig wie möglich zu modernisieren oder mich einem Zeitgeschmack anzubiedern, und gleichzeitig für meine Generation, die nach dem Krieg geborene, Akzente zu setzen, also auch nicht alles gleich wichtig zu nehmen, das für mich als wichtig bekannte aber zu betonen.

Das Bewahren einer wie auch immer definierten poetisch-sprachlichen, „dichterischen” Form stellt also interessanterweise die Voraussetzung dafür dar, den Text so bearbeiten zu dürfen, dass er einen nützlichen Erkenntnisgewinn unter zeitgenössischer Perspektive bietet. ${ }^{8}$ Im Gespräch mit Martina Hanf erklärt Peymanns Dramaturg Hermann BeIL in diesem Zusammenhang (HANF 2004: 170):

Die Balance zwischen poetischer Form und Direktheit, die in Shakespeares Zeit ihre Wirkung gehabt haben muss, das war sein Ziel.

8 Damit "normalisiert" Brasch natürlich seinen Bearbeitungsansatz. Es geht mir in diesem Aufsatz aber nicht darum, die Legitimität seiner Bearbeitung zu diskutieren, sondern sie zu beschreiben und in ihrer sozio-kulturellen Funktion zu interpretieren. Denn die Fragwürdigkeit einer Übersetzung liegt meines Erachtens nicht darin, dass sie "das Original verfälscht", sondern dass sie innerhalb der Zielkultur keine relevante Funktion besitzt. 
Um diese Beziehung zwischen poetischer Form und Direktheit (ich vermute: der Aussage) unter zeitgenössischen Bedingungen herzustellen, bildet Brasch bestimmte poetisch-rhetorische Elemente des Ausgangstextes nach. Wir werden aber sehen, dass diese sprachlich rhetorische Texttreue keineswegs „demütig” ist, sondern taktisch. Sie entspricht in ihrer Wirkung und Aussageabsicht durchaus den ebenfalls vorgenommenen Bearbeitungen der Fabel. Texttreue und Bearbeitung, Zitat und Variation dienen einer gegenwärtigen Aussageabsicht.

Entscheidend für diese Aussageabsicht und damit die Funktion der Übersetzungsstrategien ist Braschs Deutungshypothese der Thematik des Stückes. Er erklärt, dass die Fabel des Stückes eine utopische Liebesgeschichte und die darin ausgedrückten individuellen Freiheits- und Glückserwartungen angesichts der vergifteten gesellschaftlichen Verhältnisse als unmöglich darstellt - und darstellen muss, so dass bei deren letztlich unglücklichem Ende dann ,gewaltsam zusammenkommt, was gar nicht zusammenkommen will." Im Gespräch mit Martin LinZER erklärt BRASCH (LINZER 1993: 28):

Dazu kommt bei Shakespeare, mit zunehmendem Alter, immer mehr die Frage: was ist Liebe? Und er kommt immer mehr zu der Feststellung: die gibt es nicht! Da spielen zwei Geschlechter nach zwei unterschiedlichen Spielregeln miteinander, gegeneinander. Für Shakespeare eine bittere Wahrheit [...] und dem Publikum die eigene, vielleicht falsche Erkenntnis nicht vorenthaltend, daß die einfache Rechnung, wenn der Staat mörderisch ist, die Liebe es uns schon vergelten wird, nicht aufgeht. Daß die Krankheit Staat, die Krankheit Politik, die Krankheit Einsamkeit in jede Zweisamkeit, in jede Arbeit, in jedes Spiel ihre Säure gießen.

Diese zeitgenössische und unversöhnliche Interpretation der Fabel, die sich natürlich auf Textmerkmale stützen kann, verweist nicht nur auf Braschs beinahe zwanzig Jahre früher formuliertes ,großes Thema” (siehe oben),

9 So in einem Interview mit Ernst Schuhmacher in der Berliner Zeitung vom 14.03.1993. 
sondern betont, dass auch Braschs übersetzerische Tätigkeit den historischkritischen Impuls seiner gesamten künstlerischen Tätigkeit austrägt, wie und ob es ein gutes Leben in von repressivem Staat, kapitalistischer Wirtschaft und heuchlerischer Gesellschaftsmoral geprägten falschen Lebensstrukturen geben könne; vor allem wie dieser Widerspruch vom Einzelnen produktiv gestaltet werden könne.

Brasch war sich der verzerrenden Dimension seiner ,produktiven Rezeption" der Shakespeareschen Poetik bewußt, wie sich etwa an einer Strophe eines kleinen Gedichts aus der Nachwendezeit ablesen lässt, das sich in Braschs Nachlass fand (BRAscH 2002b: 30, Interpunktion so im Original):

\section{$[\ldots]$}

Der ist der größte Dichter

Ach, bleib mir, Shakespeare, treu

Wer schneidet uns Gesichter

Du mir. Ich dir. Jetzt sind wir schon Ent-2.

$[\ldots]$

Aus dem poetischen Bild des wechselseitigen Gesichter-Schneidens lese ich Braschs Erfahrung der produktiv verzerrenden Lektüre und der aneignenden, bearbeitenden Übersetzung als Erfahrung eines dialektischen Palimpsestes: dialektisch, weil Übersetzung und Ursprungstext sich gegenseitig in ihrer historischen Relativität und Andersartigkeit anerkennen und kritisieren. Als dialektisches Palimpsest ist die Übersetzung zugleich anmaßend und bescheiden. Anmaßend, weil Brasch sich und seine Zeitumstände über die historische Differenz des Ursprungstextes setzt und ihn sich aus der eigenen Sicht aneignet, und in diesem Aneignungsprozess einen Erkenntnisgewinn behauptet. Bescheiden, weil die Übersetzung die eigene historische Relativität und Vergänglichkeit bekennt. Auch sie geht entzwei und enthüllt die partielle Unzulänglichkeit und Fragwürdigkeit ihres aneignenden Bearbeitungsimpulses. 


\subsection{Die Übersetzung Wie es euch gefällt}

Im folgenden sollen nun am Text die von mir erkannten unterschiedlichen Übersetzungsstrategien gemeinsam mit den ihnen zugewiesenen politischen Funktionen dargestellt werden.

\subsubsection{Die Entscheidungen für sprachliche Texttreue: die Darstellung gesell- schaftlicher Machtstrukturen}

Brasch konstruiert eine unter sprachlichen Gesichtspunkten hybride Form, die aber über weite Strecken Kennzeichen des Ursprungstextes nachbildet. Sein Wille zu einer gewissen Texttreue, sein Wille, die „Temperatur” und „politische Poesie” des Stückes nicht zu verletzen, äußert sich rhetorisch zunächst einmal einfach darin, dass er in der Übersetzung die gereimten Zweizeiler am Szenenende wie auch den Wechsel zwischen Prosa und Blankvers beibehält. Um den Blankvers als fünfhebigen Jambus wiederzugeben, formuliert er oftmals in einer antiquierend wirkenden Syntax..$^{10}$ Der erstmalige Einsatz des Blankverses in I.2.208-218 kann dies verdeutlichen. ${ }^{11}$

DUKE FREDERICK

I would thou hadst been son to some man else, the world esteemed thy father honourable, but I did find him stille mine enemy. Thou shouldst have better pleased me with this deed hadst thou descended from another house. But fare thee well, thou art a gallant youth. I wouldst thou hadst told me of another father. [...]

ORLANDO

I am more proud to be Sir Rowland's son, his youngest son, and would not change that calling to be adopted heir to Frederick. (I.2.208-218)

\section{HERZOG}

Ich wünscht, du wärest eines andren Sohn. Die Welt hat deinen Vater sehr geehrt doch mir ist er begegnet als mein Feind. Du wärst mir lieber mit dem was du tatst, wenn du aus einem andren Hause kämst. Doch lebe wohl, du Jüngling voller Mut, ich wollt du hättest einen andren Vater. $[\ldots]$

\section{ORLANDO}

Nie wars mir lieber, Vaters Sohn zu sein, sein jüngster, unsren Namen gäb ich nie, um Erbe Herzog Fredericks zu sein. (S. 298)

10 Die Antiquiertheit hat sicherlich damit zu tun, dass durch die mehr als hundertjährige Kanonisierung der Schlegelschen Übersetzung der Blankvers in weiten Teilen der Leserschaft mit dem 19. Jahrhundert, und literarischen Modellen der deutschen Klassik und Romantik, assoziiert wird.

11 Braschs Übersetzung enthält keine Verszählung. Es werden deshalb nur die entsprechenden Seitenzahlen angegeben. Alle englischen Zitate sind der von Allan 
Wenn Duke Frederick Rosalind von seinem Hof verbannt, antwortet Rosalind in umständlicher und antiquierend wirkender Diktion:

\section{ROSALIND}

I do beseech your grace. Let me the knowledge of my fault bear with me.

(I.3.43-44)

ROSALIND

Ich bitte euer Gnaden. Gebt mir das Wissen meines Fehlers mit.

(S. 301)

Da es die Welt des Hofes ist, die im Blankvers spricht, verstärkt diese verfremdende Übersetzungsstrategie ganz im Sinne Braschs die unnatürliche Steifheit der politischen Sphäre im Stück. Die den Ursprungstext nachbildende, für heutige Ohren sprachlich verfremdende Übersetzungsstrategie dient Brasch dazu, ganz im Sinne seines großen Themas die gesellschaftlichen Bedingungen als steife, ,,versteinernde” zu kennzeichnen. Andererseits scheut Brasch nämlich nicht davor zurück, in diesen antiquierenden Blankversrhythmus doch eindeutig zeitgenössische Wörter und Strukturen einzubauen, so zum Beispiel, als Orlando auf Rosalinds Gunstbeweis stocksteif reagiert:

ORLANDO

Can I not say 'I thank you'? My better parts are all thrown down, and that which here stands up is but a quintain, a mere lifeless block. (I.2.233-235)
ORLANDO

Kann ich nicht danke sagen. Was an mir taugt, ist sehr erschöpft und der hier aufrecht steht, ist nur ein Tiger aus Papier, ein stummer Klotz. (S. 298)

Der Tiger aus Papier mag eine mehr oder wenig glückliche Formulierung sein. Auf jeden Fall ist sie eine modernisierende ${ }^{12}$ und menschlich anschauliche, mit der Orlando gerade sein momentanes, perplexes Gefangensein in falschen Formen ausdrückt. Ein erster Hinweis darauf, dass Brasch zu eigenen, aktualisierenden Strukturen greift, je individueller die Figur in einer Situation gezeichnet werden soll.

Brissenden in der Reihe The Oxford Shakespeare herausgegebenen Textausgabe von As You Like It entnommen.

12 Nach Paul (1992) und Duden Universalwörterbuch (DrosDowsKI 1989) handelt es sich bei dem Wort um eine Lehnübersetzung aus dem Chinesischen. Paul verweist auf eine 1968 übersetzte Schrift Mao Tse-Tungs als mögliche Quelle des Wortes hin. 
Mit seiner Übersetzungsweise, die politische Strukturen historisch verfremdet und die Individualität der Figuren zeitgenössisch vertraut darstellt, nutzt Brasch einfach die inhärenten Möglichkeiten des Ausgangstextes im eigenen Interpretationsinteresse. Denn diese Varianz im Register wird sowohl bei Shakespeare wie bei Brasch gerade den Figuren eingeschrieben, die alle aus der sozialen Bestimmtheit des Hofes herausfallen, nämlich Rosalind, Celia, Orlando und vor allem Touchstone. Daraus ergibt sich dann die variable Diktion, die dem Zuhörer gezielt eine historische Fremdheitserfahrung bzw. ein zeitgenössisches Vertrautheitserlebnis ermöglicht und damit politisch motivierte Sympathielenkung betreibt. ${ }^{13}$ Mittels dieser Diktion wird so der politische Zwangsrahmen des emotionalen Konfliktes betont. Es geht also bei der sogenannten Texttreue nicht in erster Linie um eine historisch genaue, um eine gegenüber der heutigen Diktion verfremdende Übersetzungsweise an sich, sondern darum, diese Übersetzungsstrategie für die politisierende Aussageabsicht des Übersetzerautors Brasch nutzbar zu machen. ${ }^{14}$

Ähnliche Taktiken lassen sich für Braschs Umgang mit deiktischen und gestischen Sprachelementen des Ausgangstextes festhalten. ${ }^{15}$ Auch hier

13 Für Verfechter der Übersetzung als Ausdruck dynamischer Äquivalenz lässt sich nebenbei festhalten, dass Braschs Text damit durchaus die seinerseits von Neuschöpfungen durchsetzte, Sprachstile klar voneinander trennende dramatische Sprache Shakespeares rhetorisch äquivalent wiedergibt. Darüber hinaus bestätigen diese Beobachtungen Nords Ansicht, dass intertextuelle Kohärenz produziert wird, wenn diese mit der Aussageabsicht (Skopos) des Übersetzers übereinstimmt.

14 Schlegels Übersetzung benutzt beispielsweise für heutige Leser durchgängig ein Register, einen antiquiert hohen Ton, so dass die sprachliche Differenzierung zwischen Hof und Wald, Höflingen und Rebellen nicht mehr gegeben ist.

15 Deiktische Sprachelemente, oder die im weiteren, Brechtschen Sinne gestischen Aspekte der Sprache, sind allgemein als jene Aspekte anerkannt, auf denen die dramatische Wirkung des Dialogs beruht. Sie definieren die Haltung der Figuren zueinander, machen die soziale Dimension der Szene deutlich, klären den dramatischen Konflikt und fördern so die imaginäre Einsicht (und/oder Identifikation) des Zuschauers in die auf der Bühne dargestellte Handlung. BASNETT (1985 und 1991), Déprats (1999) oder Hamburger (2004) haben mit unter 
dienen seine Übersetzungsentscheidungen dazu, die Konflikte des Stückes für heutige Ohren stärker hervortreten zu lassen. Einige wenige Beispiele mögen genügen, um zu zeigen, dass Brasch bei allen Aktualisierungen in der Formulierung sich genau an gestische und deiktische Einschreibungen der gesellschaftlichen Machtverhältnisse in Shakespeares Text hält. Beachten wir etwa, wie Brasch den Duktus in Orlandos Eingangsrede aktualisiert, ohne den Gestus zu verändern. Mehr noch, die Deixis wird für den heutigen Zuschauer plastischer, etwa wenn Brasch das Passiv der Eingangszeile durch eine aktive Struktur mit explizit erwähntem Subjekt ersetzt.

ORLANDO

As I remember, Adam, it was upon this fashion bequeathed me by will but poor a thousand crowns, and, as thou sayst, charged my brother on his blessing to breed me well (I.1.1ff)
ORLANDO

Adam, das schrieb unser Vater in sein Testament: Für Oliver, den ältesten, Haus, Hof und Geld, für Jaques sein Studium, und für mich 1000 lächerliche crowns, doch außerdem hat Vater meinen Bruder Oliver verpflichtet, mit seinem Erbe dafür einzustehen, daß ich so unterhalten und ausgebildet werden muß, wie sichs für unsereins gehört.

(S. 289)

In diesem Zusammenhang ist der Gebrauch des Doppelpunktes ein typisches Merkmal von Braschs Übersetzung.

\section{OLIVER}

Let me go, I say.

ORLANDO

I will not till I please. You shall hear me. My father charged you in his will to give me good education. You have trained me like a peasant, obscuring and hiding from me all gentleman-like qualities $[\ldots]$. (I.1.61-65)

\section{OLIVER}

Laß mich los, sag ich.

ORLANDO

Erst, wenn ichs will. Hör zu: Mein Vater hat dir auch vererbt: Verschaff Orlando Unterricht:

Den Knecht hast du mich lernen lassen und alle Vorzüge des feinen Herren vor mir versteckt. (S. 289)

Mit solch knappen Satzelementen und einer nebenordnenden statt unterordnenden Satzstruktur werden die Aussagen einfach kumulativ gereiht. Brasch betont durch den Doppelpunkt die im Ausgangstext angelegte ad-

schiedlichen Schwerpunkten auf die Bedeutung dieser Dimension für den Übersetzer dramatischer Texte hingewiesen, um Anschaulichkeit und dramatische Spannung zu manipulieren. 
ditive Argumentationsstruktur, setzt die gestischen Elemente damit klar voneinander $\mathrm{ab}$ und zitiert in anschaulicher Weise, wie oben bereits für die Lexik und das Versmaß herausgestellt, die im Ausgangstext angelegten Machtstrukturen. Sprachliches Material ist insofern von Bedeutung als es den von Braschs Aussageinteresse geforderten Gestus in sich trägt.

\subsubsection{Die Entscheidung für rhetorische Variationen: die poetische Verteidi- gung des Individuums in seiner Kreatürlichkeit}

Als charakteristischer, eigenständigster sprachlicher Aspekt in Braschs Übersetzung fällt einem ein spielerisch verwirrender Ton in dessen Sprache auf, der in seinen inhaltlichen Verweisen sich oftmals auf Shakespearesche Wortspiele und Anspielungen zurückverfolgen lässt, in seiner Spannung zwischen inhaltlichem Konflikt und klanglichem Fluss aber Braschs eigene Erfindung darstellt. Das formale Charakteristikum dieser Klangspiele besteht darin, dass sie semantisch Widersprüchliches lautlich einander annähern. Insbesondere wird durch körperorientierte Anspielungen (Verdauung, Sexualität) das Naturhafte und Kreatürliche an den Figuren betont, gesellschaftlich geformte Wahrnehmungsmuster werden ironisiert und entblößt.

Technisch gesehen spielt Brasch dazu oft mit Assonanzen durch homophone Silben, die sonst sinnferne Wörter ironisch emphatisch verbinden. Ein Beispiel für diesen kritischen Gebrauch von Assonanzen findet sich zum Beispiel in Orlandos Eingangsrede, in der Brasch um die Silbe „halt” herum ein widersprüchliches Wortfeld baut:

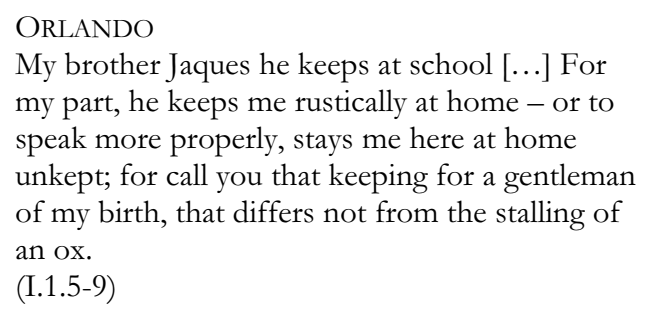

ORLANDO

Bruder Jaques kann sein Studium gut aushalten [...] aber ich muß hier wie ein Knecht haushalten. Und solchen Haushalt nennt man dann Unterhalt für einen Mann meines Stands oder besser Stalls, denn von der Stallhaltung eines Ochsen unterscheidet sich das nicht. (S. 289)

Brasch verstärkt mit seinen homophonen Wortspielen semantische Spannungen, die im Shakespeareschen Text zwischen to keep, to stay und unkept 
angelegt, aber weit weniger ostentativ ausgedrückt sind. Ähnliches gilt für den verbalen Schlagabtausch zwischen Oliver und Orlando, bei dem Brasch die Shakespearesche Alliteration in "make" und „mar” durch „zustande” und ,zuschande" bringen nicht nur nachbildet, sondern phonetisch verdichtet. Die phonetische Nähe und gleichzeitige semantische Widersprüchlichkeit geben dem Konflikt in seiner Unversöhnlichkeit noch etwas Absurdes und Groteskes.

\section{OLIVER}

Now, sir, what make you here?

ORLANDO

Nothing, I am not taught to make anything.

OLIVER

What mar you then, sir?

ORLANDO

Marry, sir, I am helping to mar that which God made, a poor unworthy brother of yours, with idleness.

(I.1.27-32)

\section{OLIVER}

$\mathrm{Na}$, Sir, was bringst du hier zustande.

ORLANDO

Ich habe nichts gelernt, um was zustande zu bringen.

OLIVER

Was machst du also zuschande, Sir.

ORLANDO

Was Gott zustande gebracht hat, mach ich zuschande: Mich selber, deinen Bruder. (S. 289/290)

Ein weiteres Beispiel für den gesellschaftskritischen Gebrauch von Alliteration und Assonanz findet sich in Szene I.2.86-99, als Rosalind, Celia, Touchstone die steife Leere im Auftreten des Höflings Le Beau (und damit des herzoglichen Hofes als Ganzes) kalauernd auf die Schippe nehmen:

Enter Le Bean

ROSALIND

With his mouth full of news.

CELIA

Which he will put on us as pigeons feed their young.

ROSALIND

Then shall we be news-crammed. [...]

LE BEAU

Fair princess, you have lost much good sport.
Auftritt LeBean

ROSALIND

Mit seinem Mund voll Neuigkeit

CELIA

Will uns damit füllen.

ROSALIND

Dann sind wir ausgefüllt mit seiner Neuigkeit. $[\ldots]$

LE BEAU

Ihr habe jede Menge Spaß verpaßt, schöne

Prinzessin. 
CELIA

Sport? Of what colour?

LE BEAU

What colour, madam? How shall I answer you?

ROSALIND

As wit and fortune will.

TOUCHSTONE

Or as the destinies decrees.

CELIA

Well said. That was laid on with a trowel.

TOUCHSTONE

Nay, if I keep not my rank -

ROSALIND

Thou loosest thy old smell.

CELIA

$[\ldots]$

LE BEAU

You amaze me, ladies. [...] (I.2.86-99)
CELIA

Sport. Ein Liebes- oder Ernstfall.

LE BEAU

Ein was. Wie soll ich darauf antworten.

ROSALIND

Im Fall der Fälle zufällig.

TOUCHSTONE

Auf jeden Fall ohne Beifall.

CELIA

Doch hoffentlich mit Einfall.

TOUCHSTONE

Aber bitte ohne Durchfall.

ROSALIND

Das ist doch nur Abfall.

CELIA

Ich kriege einen Anfall.

LE BEAU

Ihr bringt mich durcheinander, Ladies.

(S. 295)

Braschs Charakteristikum ist nicht die Intention der Szene, sondern das Mittel des kalauernden Sprachgebrauchs, der Shakespeares längst verblasste Metaphern durch erotische und fäkalische Anspielungen ersetzt. Wird in dieser Szene eine gesellschaftliche Konditionierung absurd und lächerlich gemacht, so verstärkt Braschs Übersetzung durch eben diese Assonanzen diese Wirkung auch bezüglich der individuellen, emotionalen Konditionierungen, etwa in den Liebesszenen, in denen Rosalind Orlandos naiv romantische Gefühle ironisiert. Ein kurzes Beispiel:

ORLANDO

Fair youth, I would I could make thee believe I love.

ROSALIND

Me believe it? You may as soon make her that you love believe, which I warrant she is apter to do than to confess she does. (III.2.367-371)
ORLANDO

Du schönes Junges, ich wollte, du würdest glauben, daß ich liebe.

ROSALIND

Soll ich. Mach doch die glauben, die ihr liebt, daß ihr liebt, was ihr sicher lieber wäre, zugegeben, daß sie Euch liebt.

(S. 328) 
Insbesondere Touchstone ist in diesem Zusammenhang als eine chamäleonartige Narrenfigur interessant. In I.2.50 findet sich folgende Begrüßung zwischen Celia und dem erstmalig auftretenden Touchstone:

\section{CELIA}

Peradventure $[\ldots]$ Nature $[\ldots]$ hath sent this natural for our whetstone; for always the dullness of the fool is the whetstone of the wits. How now, wit: wither wander you?

\section{TOUCHSTONE}

Mistress, you must come away to your father. (I.2.50)
CELIA

Vielleicht schickt uns der Zufall den da. Wenn uns der Witz ausgeht, zündet der uns nen anderen an. Als Zündstein sozusagen. He du, bist du ein Zündstein. [sic! Bei Brasch ohne Fragezeichen.]

TOUCHSTONE

Wenns euch gefällt, bin ich Herr Zündstein. Obwohl mir Sündschwein besser stünd. Ihr sollt zu eurem Vater kommen, Fräulein. (S. 294)

Hier wird der Narr Touchstone durch die Interpolation zu einer ambivalenten Figur, die eine kritische Spiegelfunktion übernimmt („Wenn's euch gefällt, bin ich ...”). Er wird als die Figur eingeführt, die am stärksten das kreatürliche, nach körperlichem Genuß strebende Individuum repräsentiert und sich als solches von jeder gesellschaftlichen Logik distanziert. Wenn er diese verteidigt, wie in III.2, wird er sich selbstironisch als „Herr Gründlein” einführen, ein treffend entlarvender Name für sein in diesem Gespräch bewusst spitzfindisches Gebrauchen dieser Logik. Touchstone, dessen Namen etwa Schlegel schlicht und durchaus zutreffend mit Probstein wiedergibt, wird bei Brasch zu einem vielgestaltigen sprachlichen Störer, der auf eine Weise eingeführt wird, dass er auch rhetorisch dessen Übersetzung zusammenhält: während in der sozialen Wirklichkeit die Machtstrukturen festgeschrieben sind und sich nur ihre Vertreter suchen, wird diese Erfahrungswirklichkeit im Kraftfeld seiner Sprache durcheinander gewirbelt.

Was Stephen Greenblatt in Fiction and Friction (1988) für Shakespeares Komödien festhält, dass sie nämlich auf der Bühne direkt nicht darstellbare körperliche Erregungen und Interaktionen durch sprachliche „Reibungsflächen”, insbesondere eben Ambiguitäten voller erotischer Anspielungen, ausdrücken, lässt sich auch für Braschs Übersetzung behaupten. Brasch entfaltet rhetorisch die in kompakten Shakespeareschen Metaphern angelegten Anspielungen. Damit wird eine Welt körperlicher Befriedigungen von Brasch ästhetisch auf die Bühne gebracht, 
deren klanglicher Esprit nicht nur die Lebenslust bestimmter Figuren, insbesondere Rosalinds und Touchstones, ausdrückt, sondern auch mittels identifikatorischer Rezeption die ästhetische Befriedigung realer Zuschauerwünsche anbietet.

Wie im Interview mit Linzer erwähnt, glaubt Brasch aber selbst nicht an die Möglichkeit, durch emotionale - erotische oder ästhetische - Kompensationen der Krankheit Staat und Gesellschaft zu entgehen. Damit wird die freigesetzte ästhetisch und erotische Energie aber zum Problem seines Interpretationsansatzes in dem Moment, in dem diese Figuren wieder in eine Gesellschaftsordnung sich einbinden. Dieser Moment ist im Stück die Hochzeitsszene, die gleichzeitig auch Schlussszene ist. Ohne eine ästhetische Form der Distanzierung droht die gesellschaftskritische Funktion der Sprachspiele in diesem Moment in eine vermittelnde, affirmative Utopie umzuschlagen. Shakespeare benutzt als Distanzierungsmittel metatheatralische Elemente, die aber den „Nachteil” haben, dass sie auch eine vermittelnde, versöhnende Lektüre zulassen. Es braucht uns angesichts der unversöhnlichen Deutungshypothese deshalb nicht zu wundern, dass Brasch in dieser Szene die bei Shakespeare in den Text und die Aufführungspraxis eingeschriebenen vermittelnden Elemente, vor allem metatheatralischer Art, verwirft und das von ihm zuvor betonte Lustmoment an dem blitzschnell ablaufenden und ach so ironisch wohltönenden Spiel der Signifikanten unterläuft und aushöhlt.

\subsubsection{Die Entscheidung für diskursive Verschiebungen: gegen ein wahres Leben im Falschen}

Geht man der Frage nach, wie Braschs ironische Sprachspiele sich zu Shakespeares metatheatralischen Elemente verhalten, fällt einem Braschs Bearbeitung des ersten Touchstoneschen Wortspiels auf. Seine Übersetzung bewahrt die Intentionen des Ausgangstextes, schiebt aber die politische Dimension der Metapher in den Vordergrund. Was bei Shakespeare im Hintergrund bleibt, wird hier direkt ausgedrückt. Zudem geht bereits hier eines von Shakespeares Leitmotiven verloren, nämlich die strukturierende Kraft des Wörtchens „if”. Touchstones hypothetische Struktur wird bei Brasch zu einem ironisch eingerahmten Fakt: 
ROSALIND

Ay, marry, now unmuzzle your wisdom. $[\ldots]$

TOUCHSTONE

$[\ldots]$ If you swear by that that is not, you are not forsworn. No more was this knight, swearing by his honour, for he never had any; or if he had, he had sworn it away before ever he saw those pancakes or mustard. (I.2.66-74)

ROSALIND

Ja, nimm deiner Weisheit den Maulkorb ab.

TOUCHSTONE:

Das werd ich nicht, sonst müßt ich beißen und das würd meinen Zähnen schlecht bekommen in diesem Eisenland. Doch ich versuch es so: Er hat einem Herzog die Treue geschworen und als der kein Herzog mehr war, mußte er ihm nicht treu sein und da nun ein anderer der Herzog war, hielt er eine herzögliche Treue, also war sein Schwur ehrlich.

(S. 294)

Braschs Version ist nicht nur eine drastische Beschreibung der heruntergekommenen politischen Kultur innerhalb der Fabel des Stückes, sondern auch eine politische Spitze auf die vereinigte BRD mit ihren opportunistischen Wendehälsen in Ost (aber auch West). Einmal auf diese politischen Verschiebungen aufmerksam geworden, passt es ins Bild, dass Brasch die im Ursprungstext befindlichen Hinweise auf eine andere, von Tradition und naturrechtlichen Ansätzen durchwobene Ethik eliminiert und dadurch den Ursprungstext aus seinen mittelalterlichen Verweisen herauslöst und modernisiert, so in Orlandos Anklage gegenüber Oliver (I.1.43-45) oder Rosalinds Unterscheidung zwischen den Wirkungen von Natur und Fortuna (I.2.38-45). Brasch transformiert folgendermaßen (meine Hervorhebung):

\section{ORLANDO}

$[\ldots]$ The courtesy of nations allows you $[\ldots]$ but the same tradition takes not away my blood $[\ldots]$ (I.1.43-45)

\section{ORLANDO}

„Du bist,“ sagt das Gesetz, „der Erste. Doch sagt es auch $[\ldots]^{\text {“ }}$

(S. 290)

Da Brasch der Meinung ist, dass Shakespeare zur Erkenntnis kommt, es gebe weder Liebe noch einen machtfreien Raum in der gelebten Wirklichkeit, verweigert er in seiner Übersetzung die Möglichkeit, Naturrecht und gesetztes Recht, Liebe und Lust, Ethik und Macht so aufeinander zu beziehen, dass für den Menschen eine lebbare Glückserwartung aufzufinden ist. Er modernisiert die Fabel unter neuzeitlich gesellschaftlicher Perspektive, entsprechend Adornos Diktum, dass es kein wahres Leben im Falschen gebe. Schlagend wird dies an drei Bearbeitungen innerhalb der Hochzeitsszene deutlich. Für Touchstones Schlussauftritt direkt vor den eigentlichen 
Feierlichkeiten legt Brasch eine neu gestaltete Szene vor, in der diesmal die Wortspielereien oberflächlich betrachtet eine sozial verbindende Haltung anzudeuten scheinen:

JAQUES

Can you nominate in order now the degrees of the lie? $[\ldots]$

TOUCHSTONE

[...] All these [quarrels] you may avoid $\mathrm{k}$ Lie Direct; and you may avoid that, too, an 'if'. I knew when seven justices could take up a quarrel, but when the parties v met themselves, one of them thought bi 'if', as 'if you said so and so, then I said and they shook hands and swore brothe Your 'if' is the only peacemaker; much r in 'if'.

(V.4. 84-98)
JAQUES

Zurück zu dem Duell. Was war der Grund, um den es ging und der Euch doch nichts anging.

TOUCHSTONE

Die Liebe, aber die geht Euch genauso wenig an, wie uns ihr Grund anging. [...] Denn Liebe ist kein Grund, sie ist ein Abgrund und wer duelliert sich schon über einem Abgrund. [...] Die Frage stellte sich folgendermaßen: Bring ich zuerst ihn um, dann er sie, oder zuerst sie ihn und dann er mich und schließlich wir beide sie oder umgekehrt. So beschlossen wir alle, am Leben zu bleiben. Das wollt ich sagen zum Thema Liebe und Tod.

(S. 356)

Das absurde Spiel mit den Worten und ihren Bedeutungen erhält hier entlastende (und darin scheinbar vermittelnde) Funktion, ähnlich wie in Shakespeares Text Touchstones berühmtes ,if”, das über seine metatheatralische Deutungsmöglicheit jedoch zusätzlich die utopische Hoffnung ins Spiel bringt, dass über die Lust am offen fiktionalen Spiel um Liebe im Zeichen der Macht auch ein Lustgewinn für das Spiel unter realen Bedingungen möglich sei. Betrachtet man Touchstones Monolog, verschwindet diese Möglichkeit in einem individualistischen, letztlich nihilistischen Fluchtpunkt der verwirrend absurden Sprachspielereien (warum ,schließlich”? Und was klärt die Schlussbemerkung ,oder umgekehrt”?). Hinter einem verwirrend rhetorischen Feuerwerk zeigt Touchstones Kommentar zu Liebe und Tod eine Entwertung der Liebe an. Er macht deutlich, dass entgegen erster Leseeindrücke die scheinbar befreiende Funktion dieser ironischen Sprachspiele eher als Kompensation einer sozialen Leere und einer individuellen Gleichgültigkeit zu lesen ist - und das in einem Moment des Stückes, an dem alle beteiligten Figuren auf den Beginn der Hochzeits- feierlichkeiten warten! Diese skeptische Leseweise des Shakespeareschen Schlussaktes liegt allen Interpretationen zugrunde, die die Hochzeit als ein reines Wiederherstellen patriarchaler Strukturen verstehen. In der Forschungsliteratur ist 
kontrovers diskutiert worden, ob Shakespeares Schlussszene denn eine restaurative oder subversive Wiederherstellung der patriarchal gesellschaftlichen Ordnung vollzieht. ${ }^{16}$ Braschs Version lässt diese Ambivalenz nicht mehr zu.

Weil die schönen Verwirrungen des Ardenner Waldes sich nicht in die gelebte soziale Wirklichkeit mitnehmen lassen, oder nur auf die scheinbare Weise, dass sich die Paare eben in einer zwanghaft geschönten Liebeswelt einrichten, - eine Option, die Brasch im Interview mit Martin Linzer ausdrücklich als zerstörerisch zurückgewiesen hat -, legt Brasch dem zweiten misanthropischen Hofnarren Jaques in den Mund, dass er den verbannten Herzog nun nach vollzogener Hochzeit vor die Wahl zwischen falscher Gesellschaft und wahrhafter Einsamkeit stellt:

JAQUES JAQUES

[...] So, to your pleasures;

I am for other than for dancing measures. [...]

Jeder von euch begnüg sich mit dem, der ihn betrüg, pardon, mit dem er sich vergnügt. Ich tanz nach einem Takt, der mich nicht trügt. $[\ldots]$

DUKE

HERZOG

Stay, Jaques, stay.

Bleib, Jaques, bleib.

JAQUES

To see no pastime, I. What you would have

JAQUES

I'll stay to know at your abandoned cave. (V.4.187-191)

Zum Zeitvertreib. Ich nicht. In deiner Höhle Sag was du von mir willst. Jetzt also wähle. (S. 359)

Dieser gestische Einschub verdeutlicht eine radikale Zuspitzung des Diskurses. Brasch konstruiert einen binären Wahrheitsdiskurs und betont die Unmöglichkeit, zwischen beiden Optionen, Vergnügen und potentieller (Selbst-)Täuschung einerseits und Weltflucht und potentieller Selbsterkenntnis andererseits, zu vermitteln.

Ganz deutlich wird das noch einmal im Epilog, der bei Shakespeare nicht nur einen auf patriarchale Verhältnisse verweisenden Fürsorgeauftrag an die Männer und ein Widerstandsrecht der Frauen enthält, sondern vor allem eben die Hoffnung, dass bei allen Machtunterschieden und

16 Siehe hierzu unter anderem Callaghan (2000), Dollimore (1986), Dusinberre (1975) und ERICKSON (1985). 
Mißverständnissen der Geschlechter über die gemeinsame Lust an der Fiktion Theater (als Fiktion voller Lust- und Machtimpulse) doch eine gemeinsame, eher spielerische Haltung innerhalb der gesellschaftlichen Machtkonstellation gefunden werden kann: als Lust an der Fiktion Liebe.

In beiden Texten wird die fiktionale Ebene durchbrochen und der/ die Schauspieler/in wendet sich in diesem Moment direkt ans Publikum: Die metatheatralische, offen auf Fiktionen rekurrierende Ebene und damit die ethische (Ver)handlungsfähigkeit fehlen aber in Braschs Übersetzung

\section{ROSALIND}

[...] I charge you, $\mathrm{O}$ women, for the love you bear to men, to like as much of this play as please you. And I charge you, $\mathrm{O}$ men, for the love you bear to women - as I perceive by your simpering none of you hates them - that between you and the women the play may please. If I were a woman I would kiss as many of you as had beards that pleased me, complexions that liked me, and breaths that I defied not. And I am sure, as many as have good beards, or good faces, or sweet breaths, will for my kind offer, when I make curtsy, bid me farewell.

(Epilogue)

\section{ROSALIND}

[...] Nehmt, Fraun, die ihr Männer wirklich liebt, soviel aus diesem Spiel, wies euch gefällt und ihr, Männer, die ihr wirklich Frauen liebt und eurem blöden Lächeln nach zu urteilen, gibts davon eine Menge, nehmt dieses Spiel, wies euch gefällt. Wär ich eine Frau, ich würde alle küssen, wie es mir gefällt, wär ich ein Mann, ich würde laufen, so schnell ich kann. Und wär ich beide, so ging ich aus der Welt.

(S. 359)

Gerade die beiden Schlusssätze Braschs wiederholen patriarchale Muster (die willige Frau - der bindungsunfähige Mann), über die hinaus keine Sprache oder Bilder angeboten werden (,,so ging ich aus der Welt”). ${ }^{17}$ Am Ende begrenzt die Annahme einer nicht versteinernden, sondern nun lauter falsche Bilder produzierenden Wirklichkeit die Lebens- und Imaginationsmöglichkeiten der Figur. Während der Shakespearesche Epilog das „,as you like it" als interpretatorische Herausforderung formuliert, begrenzt Braschs resignativer Schluss diese Formulierung auf eine Haltung des „,macht doch was ihr wollt, is' ja eh nur ein Irrtum."

17 In der Inszenierung schwankte Michael Maertens als Rosalind an dieser Stelle zwischen einem Knicks und einem Diener, bekam beides nicht richtig hin und betonte so visuell das unmögliche Niemandsland, in dem sich die Figur textlich am Ende befand. 


\section{Die sozio-kulturelle Motiviertheit des dialektischen Palimpsests: Kritik der Sprachspiele}

Warum tilgt Brasch das metatheatralische Spiel, wo er doch soviel Lust an sprachlichem Karneval in seine Übersetzung einschreibt? Warum hält er die darin enthaltene politische Herausforderung des Ausgangstextes nicht aus, nämlich das für neuzeitliche Augen skandalöse Angebot, wenn schon kein wahres, so doch ein gutes Leben im falschen Gesellschaftskontext führen zu können? Hier ist nicht nur auf sein großes Thema zu verweisen, sondern auch auf den konkreten sozio-kulturellen Kontext der Übersetzung und ihrer Uraufführung.

Innerhalb des Textes zeigte sich die Lust an den wirbelnden Sprachspielen als zu bannende Gefahr. Einerseits fungieren sie als gesellschaftlich subversives Korrektiv. Sobald sie aber als ästhetischer Lebensentwurf auftreten und die schlechte Wirklichkeit durch die bunte Welt der Sprachspiele verschwinden lassen - wie in Touchstones Schlussmonolog -, wird diese Lust zu einem apolitischen und damit restaurativen „Kick”; einem Kompensationsmoment, das die gleichgültige Haltung der Wirklichkeit und den Mitmenschen gegenüber nur überspielt. Braschs assonantisches Wirbeln der Signifikate bedient also zum einen gerade jenes Publikum, das sich von ästhetischen Werken primär Unterhaltung und insbesondere einen Erlebniskick erwartet. ${ }^{18}$ Der sprachlich eigenständige Teil seiner Übersetzung folgt damit einem zeitgenössischen Bedürfnis nach Kultur im Sinne einer weitgehend unpolitischen Erlebniswelt. Die Bearbeitung der Hochzeitsszene und des Epilogs verweigert sich dann aber gerade diesem Unterhaltungsbedürfnis einer postindustriellen Freizeit- und „Erlebnisgesellschaft”" ${ }^{19}$ und dem Projekt des schönen Lebens als Verlangen, etwas zu erleben. ${ }^{20}$

18 Ingeborg Pietzsch etwa beurteilt von der Inszenierung begeistert Thomas Braschs Übersetzung relativ distanzlos als „so leicht, so fröhlich, so sinnlich, daß sie mitreißt und entzückt." (Theater der Zeit, Mai/Juni 1993: 26). Der Tip, eine der beiden alternativen Berliner Stadtzeitungen, meint hingegen die Inszenierung „bedient das amüsiersüchtige Parkett. Es werden pausenlos Späßchen serviert, ohne anstrengende Interpretationskunst [...] Man bekommt viel weniger geboten, als das Stück hergeben könnte, und klatscht dafür noch Beifall."

19 So der Titel eines 1992, ein Jahr vor Braschs Übersetzung erschienenen Buches des Kultursoziologen Gerhard Schulze, in dem er die zunehmende Ästhetisierung 
Braschs doppelte Kritik der Sprachspiele, als eine gesellschaftliche Kritik durch diese und zugleich an ihnen, diskutiert unter den Bedingungen der postindustriellen bundesrepublikanischen Spaßgesellschaft das auch seinem Ausgangstext eingeschriebene Problem der sinnvollen Beziehung zwischen ästhetisch erlebter und sozial gelebter Realität. Um bei aller Lust an den entblößend kalauernden Sprachspielen nicht dieser Gesellschaft und ihrer Banalisierung von Unterhaltung in die Hände zu spielen, verwandelt Brasch die Spannung in Shakespeares Text zwischen Machtspiel und Liebesspiel, zwischen subversiver und affirmativer ästhetischer Utopie in einen unversöhnlichen Widerspruch. So wie es kein androgynes „drittes Geschlecht” gibt, gibt es auch keine Vermittlung zwischen gesellschaftlichen Machtstrukturen und ästhetischem Vergnügen.

Die meisten Rezensenten der Inszenierung von Katharina Thalbach beschrieben die Bedeutung der komödiantisch absurden Textelemente für die Aufführung und deren Wirkung. Sie freuten oder langweilten sich angesichts des Unterhaltungsangebots und nahmen es als ein eskapistisch burleskes Spektakel, letztlich also als theatralisches l'art pour l'art auf. ${ }^{21}$ Die wenigsten erkannten die gleichzeitige Problematisierung dieser Ebene. ${ }^{22}$ So

und Entpolitisierung der postindustriellen Alltagswelt und die damit einhergehenden Veränderungen in den Lebensentwürfen seiner Bürger beschreibt.

In einem anderen Text aus der Zeit spricht der Philosoph Zygmunt Bauman von "sensation-seekers and - gatherers" (BAUMAn 1998: 22).

21 Vergleiche Peter Hans Göpferts Eindruck in der Chemnitzer Freien Presse vom 17.03.93: „Ein Theater zum schnellen Konsum. Zum schnellen Lachen, zum schnellen Vergessen. Zum Nachdenken gar nicht. Damit liegt es voll im Zeitgeist.” Robin Detje: „Es ist alles aus Holz an diesem Abend. So viele Bretter, die nicht die Welt, sondern bloß Spaß bedeuten wollen. [...] Die Oberfläche als Universum." (Die Zeit, 26.03.93). Und Pietzsch begeistert sich für in der reinen Männerbesetzung sich manifestierenden „linguistic turn”: „Was ist hier echt? Was unecht? Was Natur? Was Künstlichkeit? Alles ist ein Spiel." (Theater der Zeit, Mai/Juni 1993:26).

22 Am ehesten spielt noch Gerhard Ebert in der Tageszeitung Neues Deutschland vom 16.03.93 auf dieses selbstkritische Moment an. Er erkennt einen Umschlag von Spaß in Gleichgültigkeit: „Alles ist auswechselbar. [...] Austausch des Regimes? Na und. Des Menschen Spiel um Liebe oder Täuschung, Herrschaft oder Entmachtung wird ewig, ewig währen." 
wurde die Inszenierung als Vorschlag aufgenommen, die von Brasch abgelehnte Ästhetisierung der Lebensentwürfe als bare Münze zu nehmen, oder wie Detje ablehnend formuliert: „Die Oberfläche als Universum.” Die Macht von Braschs sprachlicher Traummaschine ist so groß und verzaubernd, dass sie dem Übersetzer und seiner Deutungshypothese selbst zum Gefängnis wird. Gegen den Erwartungshorizont einer Zeit, die Bilder als Glücksversprechen produziert, versagte Braschs ironisch karnevalisierende Verweigerungsstrategie. Man kann seine Übersetzung nicht nur als partiell den Ausgangstext verwerfend (und für manche verfälschend), sondern auch als von der Zielkultur weitgehend unverstanden und somit gescheitert bezeichnen, auch wenn sie meines Erachtens innerhalb ihrer eigenen Vorannahmen sich als konsistent erweist. Solange man sich aber nur fragt, ob diese Vorannahmen dem Shakespeareschen Universum linguistisch oder kulturell adäquat sind, verpasst man die eigentliche Leistung von Braschs Übersetzung, nämlich durch ihre Mischung von ästhetischem Gewinn und Scheitern den geschichtlichen Moment zu ergreifen und begreifbar zu machen: So wie es uns heute gefällt, geht es nicht.

\section{Literaturverzeichnis}

Aaltonen, Sirkku. Time-Sharing On Stage. Drama Translation in Theatre and Society. (Topics in Translation 17). Clevedon, Multilingual Matters Ltd 2000.

BasnetT, Susan. "Ways Through the Labyrinth. Strategies and Methods for Translating Theatre Texts". In: Herman, Theo (Hg.): The Manipulation of Literature. Studies in Literary Translation. New York, St: Martin’s Press 1985, 87-102.

BasnetT, Susan. "Translating for the Theatre: The Case Against Performability". In: TTR (Traduction, Terminologie, Redaction) IV.1./1991, 99-111.

Bauman, Zygmunt. "Über den postmodernen Gebrauch der Sexualität". In: Schmidt, Gunter / StRauß, Bernhard (Hg.): Sexualität und Spätmoderne. Über den kulturellen Wandel der Sexualität, Stuttgart, Ferdinand Enke Verlag 1998, 17-35.

Benjamin, Walter. “Die Aufgabe des Übersetzers”. In: Heidermann, Werner (Hg.): Clássicos da Teoria da Tradução, Volume I, Alemão-Português. Florianópolis, UFSC (Núcleo de Tradução) 2001, 188-215. 
BRAsCH, Thomas. „Für jeden Autor ist die Welt anders: Gespräch mit Fritz J. Raddatz". In: RadDATz, Fritz J. (Hg.): ZEIT-Gespräche. Zehn Dialoge. Frankfurt am Main, Suhrkamp (st 520) 1978, 29-41.

BRAsCH, Thomas. Shakespeareübersetzungen. Mit Nachworten von Katharina Thalbach und Claus Peymann. Frankfurt/Main, Insel 2002a.

BRAsch, Thomas. Wer durch mein Leben will, muß durch mein Zimmer. Gedichte. Frankfurt am Main, Suhrkamp 2002b.

Callaghan, Dympna (Hg.). A Feminist Companion to Shakespeare. Oxford, Basil Blackwell 2000.

DÉPRATS, Jean-Michel. "Translating Shakespeare for the Theatre”. In: O'SheA, José Roberto (Hg.): Accents Now Known: Shakespeare's Drama In Translation. Ilha do Desterro. Vol. 36. 1999, 71-85.

Déprats, Jean-Michel. "Translation at the Crossroads of the Past and Present.” In: Homem, Rui Carvalho / HoenselaArs, Tom (Hg.): Translating Shakespeare for the Twenty-First Century. Amsterdam / New York, Editions Rodopi 2004, 65-78.

Detje, Robin. “Lieblingsrüpel William”. In: Die Zeit 26 Mar 1993.

Dollimore, Jonathan. "Subjectivity, Sexuality, and Transgression: the Jacobean Connection”. In: Renaissance Drama n.s. 17/1986, 53-81.

DrosDowski, Günter et al. Duden Deutsches Universalwörterbuch. Mannheim / Zürich, Dudenverlag 1989.

Dusinberre, Juliet. Shakespeare and the Nature of Women. New York, Barnes and Noble 1975.

EBERT, Gerhard. "Fünf Akte lang Anlauf genommen". In: Neues Deutschland 16. März 1993.

ERICKSON, P. Patriarchal Structures in Shakespeare's Drama. Berkeley, University of California Press 1985.

GöPfERT, Peter Hans. "Erotische Gefühle auf der Spielplatzwippe”. In: Freie Presse 17. März 1993.

Greenblatt, Stephen. „Fiction and Friction”. In: Shakespearian Negotiations. The Circulation of Social Energy in Renaissance England, Oxford: Clarendon Press 1988, 66-93. 
Hamburger, Maik. "'If it be now'. The Knocking of Fate". In: Homem, Rui Carvalho / HoenselaArs, Tom (Hg.): Translating Shakespeare for the TwentyFirst Century. Amsterdam / New York, Editions Rodopi 2004, 117-128.

Hanf, Martina. "Eine glückhafte Begegnung: Gespräch mit Hermann Beil und Jutta Ferbers". In: Hanf, Martina / Schulz, Kristin (Hg.): Das Blanke Wesen. Arbeitsbuch Thomas Basch. Berlin, Theater der Zeit 2004, 166171.

JAKOBSOn, Roman. "On linguistic aspects of translation". In: Venuti, Lawrence (Hg.): The Translation Studies Reader, London, Routledge 2000.

LinZER, Martin. "Die Flamme entsteht aus der Reibung. Gespräch mit Thomas Brasch". In: Theater der Zeit. Mai/Juni 1993, 28-31.

Nord, Christiane. Text Analysis in Translation. Theory, Methodology, and Didactic Application of a Model for Translation-Oriented Text Analysis. Amsterdam / Atlanta, Rodopi 1991.

Paul, Hermann et al. Deutsches Wörterbuch. Tübingen, Niemeyer 1992.

Pietzsch, Ingeborg. "Wunder-Wald. Berlin: Thalbachs schöner Shakespeare”. In: Münchner Merkur 15. März 1993.

SCHAPER, Rüdiger. "Stunde der Chargen”. In: Tip. Juli 1993.

SchuHmacher, Ernst. "Sich eine dünne Haut zulegen und nicht aus ihr fahren: Interview mit Thomas Brasch”. In: Berliner Zeitung 13/14. März 1993.

Schulze, Gerhard: Die Erlebnisgesellschaft. Kultursoziologie der Gegenwart. Frankfurt am Main / New York 1992.

Venuti, Lawrence. The Translator's Invisibility: A history of translation. London / New York, Routledge 1995. 